\title{
Effect of Transactional Analysis Approach Group Training on Psychological Capitals and the Temptation of Methadone- Treated Addicts
}

\author{
Masoumeh Forghani1(D), Alireza Rajaei*(D), Mohammad Hossein Bayazi1(D) \\ 1 Department of Psychology, Torbat-e Jam Branch, Islamic Azad University, Torbat-e Jam, Iran
}

*Corresponding author:

Alireza Rajaei, Department of Psychology, Torbat-e Jam Branch, Islamic Azad University, Torbat-e Jam, Iran

Tel: +989155100532

Email:rajaei.46@iautj.ac.ir

Received: 03 Sep. 2020 Accepted: 24 Oct. 2020 ePublished: 01 Nov.2021

\section{Abstract}

Background and Objective: Drug addiction is one of the health and social challenges of the present age, and psychological capital is among the protective factors in its prevention. The present study aimed to investigate the effect of Transactional Analysis (TA) approach group training on psychological capitals and the temptation of methadone-treated addicts.

Materials and Methods: This quasi-experimental study was conducted based on a pretestposttest design and a control group. The statistical population consisted of all methadonetreated addicts in addiction treatment clinics in Mashhad, Iran. Out of this population, 30 cases were randomly selected and divided into two groups of experimental $(n=15)$ and control $(n=15)$ using the random replacement method. The data were then collected through Luthans's Psychological Capitals and Wright's Craving Beliefs Questionnaires. The obtained data were analyzed in SPSS software (version 21) through univariate and multivariate covariance.

Results: The findings showed that the TA approach had a positive and significant effect on psychological capitals and its components (self-efficacy, hope, resilience, and optimism) $(\mathrm{P}<0.001)$. It also had a significant effect on reducing the temptation of the recurrence of methadone-treated addicts $(\mathrm{P}<0.001)$.

Conclusions: It can be concluded that the TA approach group training can help decrease methadone-treated addicts' psychological problems, and it can be used in counseling and addiction treatment centers.

Keywords: Addicts, Psychological capital, Temptation, Transactional analysis approach

\section{Background}

Addiction occurs when a person is physically or mentally dependent on continuous drug use over time [1]. Drug abuse kills thousands every year, destroys families and entire communities, and cripples the health care system. Opioid exposure causes the creation of long-term changes in the areas of the brain involved in reward and motivation processing and leads vulnerable individuals to seek those drugs that could be a lifelong issue [2]. The important point about reducing the incidence of high-risk behaviors and drug abuse is that this phenomenon is preventable. To prevent it, it is necessary to identify the protective factors and risk factors associated with it. One of the factors protecting against substance abuse is psychological capital [3, 4].

Psychological capitals are the perception derived from positive psychology, which includes the strengths and positive aspects of human behavior [5]. Psychological capitals are the compound and interconnected structure that includes four perceptual-cognitive components [6]. Luthans believes that when the four components of selfefficacy, optimism, hope, and resiliency are combined, they form a high-level structure called psychological capital. Each of these four components is considered a positive psychological capacity and has a valid measurement scale that is based on theory and research. It is also statedependent and growth-capable; moreover, it is significantly associated with functional consequences [7]. Research has revealed that people with high psychological capital and capacity for hope have a high ability to find alternative ways to achieve their goals in confronting obstacles; accordingly, they are less likely to turn to addictive behaviors [8]. Rabenu, Yaniv, and Elizur [9] in a study investigated the relationship of psychological capital with psychological rehabilitation and performance. The results indicated that psychological capital had a positive and direct relationship with health and performance.

Another variable proposed in the field of addiction is the temptation to recur. Temptation refers to the 
desire to use drugs. Temptation means impulses, desires, needs, or compulsions to consume and conscious experience to use drugs [10]. The greatest threat for addicts under treatment and the main factor of recurrence is the temptation, and if not controlled and recognized exactly, it can be very confusing and troublemaking for a person and may lead to drug reuse. The International Classification of Diseases has considered temptation the first criterion for diagnosing drug dependence [11]. Bandura believes that people have less ability against the temptation to use drugs when they have poor self-efficacy [12].

In recent decades, some psychotherapists have tried to use new approaches in psychotherapy. One of these approaches is the theory of Transactional Analysis (TA), which was developed and expanded by Eric Berne. Effective steps can be taken in personal cognition, treatment, and mental growth using this theory. Furthermore, it has an effective role in improving human relationships, thereby enhancing the adaptation of the individual with others and how to know oneself [13].

Berne believes that three principles constitute the general terms of the structural analysis of personality, which are phenomenologically represented as "Ego State". Ego state diagnosis, data processed ego state, and repressed ego state are called "Parent", "Mature", and "Child" in slang, respectively [14]. The "Child Ego" state is a set of emotions, attitudes, and behavioral patterns that are remnants of one's childhood. The "Parent Ego" state is a set of emotions, attitudes, and behavioral patterns that the same similar characteristics also exist in parents. The state of "Mature Ego" is described by a set of autonomous and independent emotions, attitudes, and behavioral plans that are compatible and coordinated with the existing reality [15].

Torkaman et al. [16] examined the effectiveness of TA on self-esteem and concluded that the TA group training had a significant and positive effect on self-esteem. In the same line, Etemadi et al. [17] investigated the effectiveness of TA treatment on reducing the severity of addiction and the temptation of female patients treated with methadone.

The results showed that TA group therapy had a significant effect on the temptation to use drugs and reduced temptation in methadone-treated female addicts.

\section{Objectives}

Considering the stated points and the fact that the
TA approach works on behavior, it seems that it leads to an increase in psychological capitals and a decrease in temptation recurrence. Accordingly, the main question of this research is:

Does the TA approach group training affect psychological capitals and the temptation of methadone-treated addicts?

\section{Materials and Methods}

This quasi-experimental study was conducted based on a pretest-posttest design with a control group. The statistical population included all methadone-treated addicts in addiction treatment clinics from May to July 2018. Out of this population, 30 cases were randomly selected and divided into two groups of experimental $(\mathrm{n}=15)$ and control $(n=15)$ using the random replacement method. The required data were then collected through Luthans's Psychological Capitals [18] and Wright's Craving Beliefs [19] Questionnaires. Following that, the experimental group participated in 10 two-h sessions of the TA approach training protocol; however, the control group received no training. At the end of the last session, the relevant questionnaires were administered again to the two groups. The inclusion criteria were: 1) age between 20 and 50 years, 2) male gender, 3) minimum secondary school education, 4) opium addiction, and 5) methadone-treated. On the other hand, the participants who were absent in more than two treatment sessions, and those who did not do their assignment were excluded from the study.

\section{Luthans' Psychological Capital Questionnaire}

Luthans's Psychological Capital Questionnaire was designed in 2007. This questionnaire has used standardized values that widely assess structures, such as hope, resiliency, optimism, and selfefficacy. The validity and reliability of these subscales have also been proven in previously conducted studies. This questionnaire consists of 24 questions, and each subscale consists of 6 items, which are rated on a 6-point Likert scale of "completely disagree=1", "disagree=2", "somewhat disagree $=3 "$ ", "somewhat agree $=4 "$ ", "agree $=5 "$, and "completely agree $=6 "$ ". To obtain the psychological capital score, at first, the score of each subscale is obtained separately, and then their sum is considered the total score of the psychological capital. The results of the confirmatory factor analysis indicated that this test had the factors and structures desired by the test creators. Furthermore, the results of the factor analysis confirmed the 
validity of the test construct. The Chi-square ratio of this test is equal to 24.6, and the statistics of CFI and RMSEA in this model are obtained at 0.97 and 0.08 , respectively [4]. Moreover, in a study performed by Khosroshahi et.al, the reliability ratio of this questionnaire was determined at 0.85 based on Cronbach's alpha [20].

The Craving Beliefs Questionnaire was also utilized in this study to collect data. This questionnaire has 20 questions designed by Wright (2003), and it aims to measure the temptation ratio to use drugs. To obtain the total score of the questionnaire, the total scores of all questions are added together. These scores will have a range between 20 and 140. Higher scores will indicate a greater temptation of the respondent to use drugs and vice versa. This test has good validity and reliability; in addition, its reliability has been reported to be 0.95 using Cronbach's alpha coefficient. Furthermore, its face and content validity have been confirmed by relevant professors and experts [21].

The methadone-treated addicts in the experimental group were requested to participate in 10 two-h sessions of TA training. The TA training protocol is presented in Table 1 .

Table 1. Transactional Analysis Training Protocol

\begin{tabular}{|c|c|}
\hline Sessions & Content \\
\hline Session 1 & $\begin{array}{c}\text { Being familiar with the group and goals; being familiar with the symptoms after quitting substances; teaching } \\
\text { personality structure in terms of TA (parent-mature-child); responding to three questions: Who am I? How do I } \\
\text { behave? Why am I behaving like this? }\end{array}$ \\
\hline Session 2 & $\begin{array}{l}\text { How to draw a diagram of self-states or Ego Gram; teaching (supportive parent and normal child) healthy and } \\
\text { unhealthy parenting styles (controlling and blaming parent, as well as obedient, rebellious, and isolated child). }\end{array}$ \\
\hline Session 3 & $\begin{array}{r}\text { Teaching inefficient and repetitive behaviors (internal and external barriers, sick parent or sick child); teaching } \\
\text { different types of relationships (parallel, cross, hidden) }\end{array}$ \\
\hline Session 4 & Teaching the topic of caresses and drawing a diagram of the caresses bank of each person. \\
\hline Session 5 & Teaching different types of behaviors, activities, pastimes, intimacy, occasions, and isolation. \\
\hline Session 6 & Teaching psychological games and giving exercise in the session. \\
\hline Session 7 & Healing the inner child and teaching its techniques. \\
\hline Session 8 & $\begin{array}{l}\text { Challenging members and finding appropriate and mature solutions to solve the challenge by group members } \\
\text { to activate mature, as well as increase resiliency and efficiency (practice in session). }\end{array}$ \\
\hline Session 9 & $\begin{array}{l}\text { Challenging members and finding appropriate and mature solutions to solve the problem by group members to } \\
\text { activate mature and increase optimism and hope (practice in the session). }\end{array}$ \\
\hline Session 10 & Reviewing previous sessions, questions, and answers; administering the post-test. \\
\hline
\end{tabular}

Frequency tables and charts, as well as central indices and distribution indices (i.e., mean $\pm \mathrm{SD}$ ), were calculated in the descriptive statistics section. In addition, the multivariate analysis of covariance was used in the inferential statistics. The abovementioned statistical analyzes were performed in SPSS software (version 21).

\section{Results}

This study was conducted on 30 cases who were divided into experimental $(\mathrm{n}=15)$ and control groups $(n=15)$ with the mean ages of $38.73 \pm 88.66$ and $34.73 \pm 7.50$, respectively. As can be observed, the two groups are homogeneous in terms of age. Table 2 tabulates the descriptive indicators of the data obtained from the pretest and posttest of psychological capital. Results reveal that the calculated equality of variances (Levene's F) at the level of $\mathrm{P}>0.05$ is not significant; accordingly, there is no significant difference between the experimental and control groups at the pretest regarding the variances of the scores of this scale. The results also showed that the Z-KolmogorovSmirnov was not significant for the tested variables in both groups ( $\mathrm{P}>0.05)$; therefore, it can be concluded that the scores have been normal, and the presupposition of using covariance has also been observed. Furthermore, the test of variance-covariance homogeneity hypothesis (Box's $\mathrm{M}=7.93, \quad \mathrm{~F}=0.67, \quad \mathrm{Sig}=0.75$ ) indicated that the significance has been higher than 0.05 , which means that equality exists in the observed covariance matrices of the quantitative variables of each factor. Multivariate analysis of covariance showed a significant effect for the treatment method factor $(\mathrm{P}<0.001)$. Table 3 shows the results of the univariate analysis of covariance in the text of multivariate analysis of covariance for each of the test cases on methadone-treated addicts.

As can be observed in Table 3 , there is a significant difference between the experimental and control groups in terms of self-efficacy $(\mathrm{F}=349.09, \mathrm{P}<0.001)$, hope $(\mathrm{F}=120.69, \mathrm{P}<0.001)$, resiliency $\quad(\mathrm{F}=217.96, \quad \mathrm{P}<0.001), \quad$ optimism $(\mathrm{F}=180.20, \quad \mathrm{P}<0.001), \quad$ psychological capital $(\mathrm{F}=21.89, \quad \mathrm{P}<0.001)$, and temptation $(\mathrm{F}=60.38$, $\mathrm{P}<0.001)$. 
Table 2. Descriptive Indicators of Pretest-Posttest Data of the Tested Scales

\begin{tabular}{|c|c|c|c|c|c|c|}
\hline Variable & Stage & Group & Mean & Standard Deviation & Minimum & Maximum \\
\hline \multirow{4}{*}{ Self-Efficacy } & \multirow{2}{*}{ Pretest } & Experimental & 27 & 6.98 & 15 & 36 \\
\hline & & Control & 28.4 & 3.74 & 20 & 32 \\
\hline & \multirow{2}{*}{ Posttest } & Experimental & 29.67 & 4.7 & 24 & 36 \\
\hline & & Control & 28 & 3.46 & 22 & 34 \\
\hline \multirow{4}{*}{ Hope } & \multirow{2}{*}{ Pretest } & Experimental & 25.13 & 6.37 & 14 & 36 \\
\hline & & Control & 25.93 & 5.51 & 18 & 33 \\
\hline & \multirow{2}{*}{ Posttest } & Experimental & 27.6 & 5.26 & 19 & 36 \\
\hline & & Control & 25.47 & 5.59 & 17 & 33 \\
\hline \multirow{4}{*}{ Resiliency } & \multirow{2}{*}{ Pretest } & Experimental & 22.87 & 2.99 & 19 & 31 \\
\hline & & Control & 23.93 & 3.61 & 19 & 31 \\
\hline & \multirow{2}{*}{ Posttest } & Experimental & 26.07 & 4.27 & 20 & 34 \\
\hline & & Control & 23.4 & 3.6 & 20 & 31 \\
\hline \multirow{4}{*}{ Optimism } & \multirow{2}{*}{ Pretest } & Experimental & 23.4 & 2.53 & 19 & 28 \\
\hline & & Control & 22.27 & 3.77 & 17 & 30 \\
\hline & \multirow{2}{*}{ Posttest } & Experimental & 28.6 & 3.79 & 24 & 38 \\
\hline & & Control & 22.27 & 4.17 & 16 & 31 \\
\hline \multirow{4}{*}{ Psychological Capital } & \multirow{2}{*}{ Pretest } & Experimental & 96.93 & 19.24 & 52 & 126 \\
\hline & & Control & 101 & 12.17 & 82 & 123 \\
\hline & \multirow{2}{*}{ Posttest } & Experimental & 111.93 & 13.86 & 91 & 139 \\
\hline & & Control & 99.13 & 10.99 & 81 & 117 \\
\hline \multirow{4}{*}{ Temptation } & \multirow{2}{*}{ Pretest } & Experimental & 76 & 27.63 & 33 & 138 \\
\hline & & Control & 82.87 & 17.66 & 49 & 105 \\
\hline & \multirow{2}{*}{ Posttest } & Experimental & 53.93 & 17.85 & 22 & 77 \\
\hline & & Control & 84.47 & 16.66 & 52 & 106 \\
\hline
\end{tabular}

Table 3. Results of the multivariate analysis of covariance

\begin{tabular}{|c|c|c|c|c|c|c|}
\hline Variables & SS & $\mathrm{df}$ & MS & $\mathrm{F}$ & Sig. & Eta \\
\hline Self-Efficacy & 3338.37 & 1 & 3338.37 & 349.09 & 0.001 & 0.89 \\
\hline Hope & 2466.32 & 1 & 2466.32 & 120.69 & 0.001 & 0.77 \\
\hline Resiliency & 2874.58 & 1 & 2874.58 & 217.96 & 0.001 & 0.81 \\
\hline Optimism & 2904.41 & 1 & 2904.41 & 180.24 & 0.001 & 0.80 \\
\hline Psychological Capital & 3472.4 & 1 & 1736.2 & 21.89 & 0.001 & 0.62 \\
\hline Temptation & 12532.59 & 1 & 6266.29 & 60.38 & 0.001 & 0.82 \\
\hline
\end{tabular}

\section{Discussion}

The present study was conducted to evaluate the effectiveness of the TA approach group training on psychological capitals and the temptation of methadone-treated addicts. The results showed an increase in the psychological capital and a decrease in the temptation to consume substances among the methadone-treated addicts in the experimental group, compared to the control group. Therefore, it can be concluded that the TA training program can change these variables and can be used in clinical and treatment environments, as well as addiction treatment centers. This finding is in line with the results obtained from previously conducted studies by Torkaman et al. [16] and Etemadi et al. [17].

Torkaman et al. [16] found that the TA treatment had a positive effect on reducing substance consumption temptation in methadone-treated female addicts. Similarly, Rabenu et al. [9] showed that psychological capital led to positive health and performance. According to the results of a study carried out by Etemadi et al., the TA reduced the severity of addiction in methadonetreated addicts [17].

In explaining these findings, it can be said that
Eric Berne's analytical model includes the states of self, interaction, caresses, first drafts of life, everyday situations, and time organizing. The purpose of the TA approach is to identify specific types of interactive situations that help the patient recover from rehabilitation. It also aims to increase the patients' awareness of the responsibilities associated with their actions and behaviors [18].

The TA group training helps methadone-treated addicts to quit substance abuse by being aware of mental states, communication principles and roles, self-expression method, caressing, complementary and reciprocal relationships, involuntary restorations, basic living conditions, and the importance of trust in families. Moreover, the TA group therapy can positively increase a person's self-awareness about the interpersonal relationship by influencing the factor of temptation to consume substances [19].

Methadone-treated addicts by participating in the TA training group were able to help themselves in interpersonal relationships by better understanding the real self and planning appropriate communication methods and overcoming the temptation to use drugs and recurrence. In addition, based on the TA approach, the pleasurable and 
rewarding parts of drug abuse were reduced. "Ego" states are experienced in role-playing and recognizing inappropriate life games; moreover, they help people determine a new plan for a new life and end their addiction forever.

Regarding the limitations of the study, one can refer to the fact that the participants in this study were male addicts. Moreover, there was no time to follow up the evaluation results, and it was no possibility to hold sessions for the families of addicts.

\section{Conclusions}

It can be concluded that the TA approach group training can help decrease methadone-treated addicts' psychological problems; moreover, it can be used in counseling and addiction treatment centers.

\section{Ethical Considerations}

Compliance with ethical guidelines

All ethical principles were considered in this study. The participants were informed about the research objectives and procedures. Informed consent was obtained from the participants, and they were assured of the confidentiality of their information. Moreover, the subjects were free to withdraw from the study if desired. They were also informed that they would be provided with the results of the research.

Funding

This study received no specific grant from funding agencies in the public, commercial, or not-for-profit sectors.

\section{Authors' contributions}

Conceptualization [Masoumeh Forghani]; Methodology [Alireza Rajaei]; Investigation [Mohammad Hossein Bayazi]; Writing-Original Draft [Masoumeh Forghani]; WritingReview \& Editing, Author names [all author]; Funding Acquisition, [all author]; Resources, [all author]; Supervision, [Alireza Rajaei]

Conflict of interest

The authors declare that they have no conflict of interest.

\section{Acknowledgments}

The authors would like to thank all participants, who greatly cooperated in this study.

\section{References}

1. Browne CJ, Godino A, Salery M, Nestler EJ. Epigenetic mechanisms of opioid addiction. Biological Psychiatry. 2020; 87(1):22-33. [DOI:10.1016/j.biopsych.2019.06.027] [PMID] [PMCID]

2. Luthans F, Avolio BJ, Avey JB, Norman SM. Positive psychological capital: measurement and relationship with performance and satisfaction. Personnel Psychology. 2007; 60(3):541-72. [DOI:10.1111/j.1744-
[D. 6570.2007.00083.x]

3. van Wyk R. The manifestation of familiness resources and psychological capital as familiness capital: A conceptual analysis. International Business \& Economics Research Journal (IBER). 2013; 12(9):1021-40. [DOI:10.19030/iber.v12i9.8050]

4. Lee Ch, Chu KK. Understanding the effect of positive psychological capital on hospitality interns 'creativity for role performance. International Journal of Organizational Innovation (Online). 2016; 8(4):213.
5. Rahimi H, Aghababaei R. The relationship between social capital and psychological capital in female students of Kashan University. Woman and Family Studies. 2018; 10(39):7-22.

6. Rashidi AR, Hojat Khah M, Rasouli A, Jami M. Causal relationship between addiction potential through early maladaptive schemas, psychological capital and basic psychological needs mediated family communication patterns. Research on Addiction. 2017; 10(40):45-64.

7. Rasooli R, Sadeghian M. On the effectiveness of training optimistic attributional styles in reducing addiction potential and change into students' attributional styles. Scientific Quarterly Research on Addiction. 2016; 10(38):25-40.

8. Afshary M, Babakhani N, Ahmadi A. Comparison of the rate of self-esteem, self-efficacy \& self-regulation in opiates \& stimulant substance dependents with normal people. Scientific Journal of Forensic Medicine. 2016; 22(1):15-22.

9. Rabenu E, Yaniv E, Elizur D. The relationship between psychological capital, coping with stress, well-being, and performance. Current Psychology. 2017; 36(4):875-87. [DOI:10.1007/s12144-016-9477-4]

10. Bastami AR, Dalvandi A, Khankeh HR, Bastami M. The effect of resiliency skills training on attitude toward substance abuse in secondary school students of Khorramabad. Iranian Journal of Rehabilitation Research. 2016; 2(3):40-8.

11. Krasikova DV, Lester PB, Harms PD. Effects of psychological capital on mental health and substance abuse. Journal of Leadership \& Organizational Studies. 2015; 22(3):280-91. [DOI:10.1177/1548051815585853]

12. Bandura, A. Social learning theory cognitive therapy and emotional disorders. New York; International University Press; 1999

13. Weiland BJ, Nigg JT, Welsh RC, Yau WY, Zubieta JK, Zucker RA, et al. Resiliency in adolescents at high risk for substance abuse: flexible adaptation via subthalamic nucleus and linkage to drinking and drug use in early adulthood. Alcoholism: Clinical and Experimental Research. 2012; 36(8):1355-64. [DOI:10.1111/j.15300277.2012.01741.x]

14. Berne E. Transactional Analysis. Trans: Fassih E. Tehran: Farhang Nashrno; 2018.

15. ShafiAbadi A. Theories of counseling and psychotherapy. Tehran: University Publication Center; 2005.

16. Torkaman M, Farokhzadian J, Miri S, Pouraboili B. The effect of transactional analysis on the self-esteem of imprisoned women: a clinical trial. BMC Psychology. 2020; 8(1):3. [DOI:10.1186/s40359-019-0369-x] [PMID] [PMCID]

17. Etemadi-Chardah N, Matinpour B, Heshmati R. Effectiveness of transactional analysis group therapy on addiction intensity of woman patients treated with methadone. Addiction \& Health. 2017; 9(3):146. [PMID] [PMCID]

18. Naghibi H, Khazaei O, Khazaei F, RanjbarSudejani Y. On the effectiveness of group therapy with transactional analysis (TA) approach in the reduction of addiction severity among female patients under methadone treatment. Scientific Quarterly Research on Addiction. 2017; 11(41):281-98.

19. Tangolo AE, Massi A. A contemporary perspective on transactional analysis group therapy. Transactional Analysis Journal. 2018; 48(3):209-23. [DOI:10.1080/03621537.2018.1471288]

20. Khosroshahi JB, Nosratabadi TH, Bayrami M. The relationship between psychological capital and personality traits with job satisfaction among librarians' in public libraries in Tabriz. Pajoohandeh Journal. 2013;17(6):313-9

21. Saedy M, Ardani AR, Kooshki S, Firouzabadi MJ, Emamipour S, Mahboub LD, Mojahedi M. Effectiveness 
of acceptance-commitment therapy on craving beliefs in patients on methadone maintenance therapy: a pilot study. Journal of Rational-Emotive \& Cognitive-Behavior Therapy. 2018;36(3):288-302. 